\title{
Prácticas de laboratorio alineadas con el diseño de un proyecto de investigación mediante metodología docente ABP: el proyecto Agua de Fagos.
}

\author{
Isaias Sanmartín ${ }^{\text {y }}$ Ana Lloret ${ }^{\text {b }}$
}

a. Facultad de Biotecnología. Universidad Católica de Valencia b. Departamento Fisiología. Facultad de Medicina. Universidad de Valencia

\begin{abstract}
Resumen
Las prácticas de laboratorio académicas no están, en general, diseñadas para desarrollar las competencias relacionadas con el trabajo independiente del alumno. En el presente artículo proponemos una nueva forma de diseñar prácticas experimentales, alineando el desarrollo de un proyecto de investigación escrito con la realización posterior del proyecto a nivel práctico en el laboratorio, en un poderoso ensamble que genera aprendizajes muy interesantes para desarrollar las competencias relacionadas con la autonomía del alumno en el laboratorio experimental. La metodología utilizada es el aprendizaje basado en problema $(A B P)$.
\end{abstract}

Keywords: ABP, aprendizaje basado en problemas, competencias, metodologías docentes, estrategias docentes

\section{Introducción}

Existe una dicotomía o separación entre el modo de proceder del alumno en las prácticas de laboratorio universitarias y el modo en que se trabaja en la realidad a nivel profesional. Las prácticas de laboratorio académicas no están, en general, diseñadas para el trabajo independiente del alumno. El profesor proporciona una serie de protocolos de laboratorio y realiza una demostración de cómo se maneja el instrumental y reactivos para obtener el resultado experimental. El alumno debe repetir los pasos que ha visto realizar al profesor, o que le vienen indicados muy detalladamente en el manual de prácticas proporcionado. Si le surge alguna duda o una de las operaciones es delicada, el instructor está presente para aclararlo o ayudar en la realización de la práctica. Habitualmente también, la serie de prácticas de una asignatura está diseñada para que cada práctica sea independiente, realizándose en única sesión y sin relación entre ellas. Cada práctica comienza y acaba en una misma sesión, produciendo un resultado experimental que no guarda relación con las prácticas de las siguientes sesiones.

De esta manera, la actividad que realizan los alumnos en las sucesivas prácticas a lo largo del Grado es muy distinta a la que se encontrarán cuando deban trabajar de manera independiente en una situación profesional real.

El profesional que acomete un proyecto no suele disponer de protocolos, sino que debe obtenerlos en la bibliografía científica. Además, deben adaptar el protocolo a sus experimentos y a la coyuntura de su laboratorio. Es habitual también que la trayectoria 
o vía experimental para conseguir los objetivos del proyecto no sea única, sino que el profesional debe elegir entre varios caminos posibles, incluyendo la selección adecuada de los protocolos o técnicas de entre las varias disponibles que son aplicables. Debe existir una planificación, lo que llamamos un "proyecto de investigación”, en la que se imbriquen de forma clara y lógica la secuencia experimental y protocolos seleccionados, técnicas, reactivos e instrumental de laboratorio que se va a utilizar para llevarlo a cabo. Esta es otra competencia profesional paralela a la anterior, la capacidad de planificar un proyecto de investigación o desarrollo de un producto, a partir del objetivo solicitado en la empresa o centro de investigación.

Como resultado de esta separación entre la actividad profesional real y la experiencia del alumno a lo largo de sus actividades prácticas académicas, los alumnos egresan de su titulación con una serie de carencias que impactan muy negativamente en su desempeño en el ámbito profesional real. Dicho de otra forma, carecen de las competencias necesarias para funcionar adecuadamente a nivel profesional.

La presente propuesta utiliza la metodología ABP ("aprendizaje basado en problemas") para diseñar unas prácticas enfocadas a la realidad (Albanese, 1993; Duch, 2001). El alumno es sometido a una experiencia de aprendizaje cooperativo ABP 4x4 (Prieto., 2006) en la que diseñará un proyecto de investigación a nivel teórico que luego tendrá oportunidad de desarrollar en el laboratorio de manera independiente, sin la ayuda o supervisión cercana del profesor, tal como es requisito de la metodología ABP.

\section{Objetivos}

- Desarrollar la competencia en los estudiantes para trabajar de manera independiente en el laboratorio experimental

- Desarrollar la competencia en los estudiantes para el trabajo en equipo en proyectos de I\&D biotecnológicos

- Desarrollar la competencia en los estudiantes para el diseño de proyectos de I\&D biotecnológicos

\section{Desarrollo de la innovación}

Las prácticas se realizan en la asignatura "Virología" de 3er curso de la Facultad de Biotecnología de la Universidad Católica de Valencia.

El problema ABP planteado es el siguiente:

Pertenecéis al equipo de I\&D de una empresa biotecnológica especializada en productos para la industria alimentaria. Se os pide que desarrolléis un nuevo proyecto: el "agua de fagos". Se trataría de un desinfectante biológico, no químico, para responder a la solicitud de una empresa multinacional de hamburgueserías de un agente eficaz que no altere las características organolépticas de sus productos, ya que han tenido problemas de contaminación de sus productos cárnicos con cepas enterotoxigénicas de E. coli y otros coliformes. El agua de fagos contendría virus líticos, y se utilizaría pulverizando una pequeña cantidad del líquido sobre las piezas de carne, eliminando las bacterias de la superficie en caso de que estuviesen contaminadas. 
Cita: "La cepa de Escherichia coli O157: H7 (E. coli O157: H7) puede causar enfermedades graves. Ha causado varios brotes importantes de intoxicación por alimentos. Se estiman en unos 73.000 los casos reportados en los Estados Unidos cada año. El microbio se transmite a través de alimentos contaminados, tales como carne de hamburguesa, huevos y las frutas y verduras sin lavar."

Las piezas de carne picada están compuestas de una mezcla de carnes de cerdo y pollo y algunas variedades también contienen una cierta cantidad de huevo. Disponéis en vuestro laboratorio de dos cepas de bacterias, E. coli B y E. coli MM294, que usaréis como modelo experimental, representando las cepas enterotoxigénicas que habitualmente se encuentran como contaminantes del producto cárnico. Utilizalas en los experimentos para diseñar el producto "agua de fagos" que tu empresa te ha solicitado. Los bacteriófagos para diseñar el producto "agua de fagos" los capturaréis a partir de muestras de campo procedentes de granjas de animales a partir de los que se obtiene la carne.

Los alumnos se distribuyen en grupos de 4 miembros, que realizarán juntos tanto el proyecto teórico escrito como el ulterior proyecto práctico en el laboratorio. En las 4 semanas siguientes, desarrollarán el proyecto teórico según las 4 fases de la metodología ABP $4 \times 4$ de Prieto y colaboradores. Cada semana el grupo de alumnos escogerá un nuevo "líder o secretario de grupo" que dirigirá la actividad, dando así la oportunidad a todos de actuar como líderes de grupo. Para el proyecto práctico, los alumnos escogerán a un único director de proyecto o investigador principal, que dirigirá a sus compañeros y tendrá la última palabra en cuanto a las decisiones a tomar en el laboratorio sobre el trabajo experimental del proyecto.

\section{El proyecto consta de dos fases:}

Una primera fase "teórica" en las que los alumnos diseñarán en su grupo de trabajo (4 alumnos) el proyecto de investigación, entregando un informe escrito y una presentación con diapositivas PowerPoint; realizando una subsiguiente sesión de exposiciones orales en la que presentarán su proyecto y se enfrentarán a un turno de preguntas y debate con el resto de grupos que también presentan sus proyectos. Esta fase se realiza a lo largo de 4 semanas mediante la metodología ABP $4 \mathrm{x} 4$, y al concluir deben entregar un informe escrito y una presentación PowerPoint sobre el proyecto realizado. Para los debates, aporte de ideas e intercambio de opiniones, se prepararon una serie de 4 foros de opinión mediante la plataforma Moodle, en la que los alumnos debían discutir la actividad con sus compañeros y realizar sus contribuciones y aportaciones al trabajo en forma escrita. La evaluación de esta parte consta de 3 elementos: el informe escrito, la exposición oral del PowerPoint y la "defensa" realizada del propio proyecto en el turno de preguntas tras la exposición oral.

Una segunda fase "práctica" en la que cada grupo desarrollará su propio proyecto en el laboratorio a lo largo de 6 sesiones de 2 horas de duración. Esta fase se evalúa mediante la entrega de resultados experimentales (sin tener en cuenta más que se haya realizado el experimento correspondiente, ya que se asume que son alumnos principiantes que cometerán numerosos errores) y mediante un examen de tipo test que demuestre que se han entendido bien las técnicas y métodos utilizados en el proyecto. 


\section{Los materiales proporcionados a cada grupo de alumnos son los siguientes:}

Para el desarrollo del proyecto de investigación "teórico", se proporciona a los estudiantes un documento llamado "Técnicas a emplear en el proyecto agua de fagos", que contiene descritos una selección de protocolos y métodos de laboratorio suficientes para desarrollar el proyecto, seleccionadas de dos trabajos de tesis doctorales (DINI, 2011; SPRICIGO, 2012):

Técnicas generales

Aislamiento de bacteriófagos desde muestras de campo

Cinética de adsorción fágica

Cinética de infección fágica

Experimentos para comprobar la estabilidad fágica en diferentes condiciones ambientales $\left(\mathrm{pH}, \mathrm{T}^{\mathrm{a}}\right)$

Parámetros del ciclo biológico fágico (one-step growth)

Purificación y obtención de lisados fágicos

i) Lisado en medio líquido

ii) Lisado en placa

Test de la gota

Titulación de fagos mediante ensayo de punto final

Titulación de lisados fágicos

Técnicas de biología molecular

Extracción de ADN

Restricción del ADN fágico

Electroforesis convencional en gel de agarosa

Secuenciación del ADN

Análisis bioinformáticos del ADN fágico

Este recopilatorio de técnicas y protocolos representan tan sólo algunas de las posibles técnicas a emplear entre las múltiples alternativas o trayectorias experimentales posibles para realizar el proyecto, pero es suficiente para diseñarlo de forma casi completa. No están ordenados ni es necesario utilizarlos todos para diseñar el proyecto, pero representan un buen punto de partida para que los alumnos debatan ideas, estudien los protocolos y puedan comenzar la elaboración del proyecto. Algunas de las técnicas y la secuencia de pasos lógicos necesarios para acometer el proyecto no se encuentran en este documento, y deberán ser buscadas por los alumnos en otras fuentes de información o debatirlas en su grupo y proponer soluciones por sí mismos.

Para el desarrollo "práctico" del proyecto en el laboratorio, se proporciona a cada grupo de estudiantes:

- un ejemplar del libro Bacteriophagues (Kutter E, Sulakvelidze A, 2004), en cuyo apéndice "Working with Bacteriophages: Common Techniques and Methodological 
Approaches" de 58 páginas se recogen los protocolos de laboratorio y métodos principales del trabajo específico con bacteriófagos.

- 12 muestras diferentes de tierra* obtenidas de recintos donde se encuentran animales estabulados (pollo, cerdo, caballo) en las que presumiblemente se encontrarán bacteriófagos

- medios de cultivo de bacterias, reactivos y material fungible (pipetas Pasteur, placas Petri, tubos eppendorf, tubos de centrífuga, etc.), e instrumental del laboratorio microbiológico (autoclave, instrumental de vidrio, micropipetas volumétricas, ultracentrífugas, cubetas de electroforesis, espectrofotómetro de absorción, mechero Bunsen, incubador con agitación, estufas de cultivo bacteriano, cabina de seguridad biológica, etc.)

- una cepa de Escherichia coli inofensiva, la cepa E.coli B, que sustituye a las bacterias protagonistas del proyecto (E.coli enterohemorrágica O157:H7), ya que por su peligrosidad sería arriesgado utilizarla durante las sesiones prácticas

- carne picada de pollo o cerdo

*algunas de las muestras se adicionan previamente por el profesor de colifago T4, para asegurar el que sea posible encontrar al menos un fago para lisar la cepa E.coli $B$ que se suministra en las prácticas

A partir de estos materiales, cada grupo debe trabajar de forma independiente, organizar su propio trabajo, y desarrollar las experiencias a lo largo de las diferentes sesiones según decidan. La trayectoria o vía elegida para desarrollar el proyecto se deja libre a la decisión de los estudiantes.

\section{Resultados}

Los alumnos fueron consultados mediante una encuesta de evaluación voluntaria sobre las prácticas ABP realizadas. De los 48 alumnos matriculados, 33 respondieron la encuesta. Los resultados son los siguientes.

PREGUNTA $n^{\circ} 1$. La actividad de plataforma "proyecto agua de fagos" se desarrolla en grupo y pretende desarrollar competencias relacionadas con las habilidades de relación personal con los compañeros de trabajo y el trabajo en grupo ("Capacidad crítica y autocrítica", "Capacidad de trabajar en equipo" y "Habilidades de relaciones interpersonales").

Tabla 1. Resultados de la pregunta $\mathrm{n}^{0} 1$

\begin{tabular}{lc}
\hline ¿Consigue el objetivo pretendido? & \% de estudiantes que eligen esta respuesta \\
\hline Lo consigue completamente & 33,33 \\
Lo consigue en su mayoría & 42,42 \\
Lo consigue sólo parcialmente & 18,18 \\
No lo consigue & 6,06 \\
\hline
\end{tabular}

PREGUNTA $n^{\circ} 2$. El intercambio del rol de secretario de grupo cada semana durante la actividad de plataforma "proyecto agua de fagos" pretende dar oportunidades de desarrollar las competencias "Liderazgo" e "Iniciativa y espíritu emprendedor". 
Prácticas de laboratorio alineadas con un proyecto de investigación mediante ABP: el proyecto Agua de Fagos

Tabla 2. Resultados de la pregunta $\mathrm{n}^{\circ} 2$

\begin{tabular}{lc}
\hline ¿Consigue el objetivo pretendido? & \% de estudiantes que eligen esta respuesta \\
\hline Lo consigue completamente & 21,21 \\
Lo consigue en su mayoría & 21,21 \\
Lo consigue sólo parcialmente & 39,39 \\
No lo consigue & 18,18 \\
\hline
\end{tabular}

PREGUNTA n ${ }^{\circ}$ 3. La actividad de plataforma "proyecto agua de fagos" se desarrolla por medio de mensajes escritos en plataforma y pretende desarrollar competencias como "Comunicación escrita en la propia lengua", "Capacidad de análisis y síntesis" y "Que los estudiantes puedan transmitir información, ideas, problemas y soluciones a un público tanto especializado como no especializado".

Tabla 3. Resultados de la pregunta $\mathbf{n}^{0} 3$

\begin{tabular}{ll}
\hline ¿Consigue el objetivo pretendido? & $\%$ de estudiantes que eligen esta respuesta \\
\hline Lo consigue completamente & 18,18 \\
Lo consigue en su mayoría & 24,24 \\
Lo consigue sólo parcialmente & 36,36 \\
No lo consigue & 21,21 \\
\hline
\end{tabular}

PREGUNTA $n^{\circ}$ 4. La actividad de plataforma "proyecto agua de fagos" está diseñada para desarrollar una serie de competencias transversales asociadas a la asignatura tales como:

- Habilidad de la gestión de la información (habilidad para buscar y analizar información procedente de fuentes diversas)

- Habilidades básicas del manejo del ordenador relacionadas con el ámbito de estudio

- Toma de decisiones, capacidad de análisis y síntesis

- Capacidad de organización y planificación

- Comunicación oral y escrita en la propia lengua

- Capacidad de trabajar en equipo inter. y multidisciplinar

- Habilidades de relaciones interpersonales

- Capacidad crítica y autocrítica

- Capacidad de aprender

- Capacidad de adaptarse a nuevas situaciones

- Capacidad de generar nuevas ideas (creatividad)

- Liderazgo, iniciativa y espíritu emprendedor

- Capacidad de aplicar los conocimientos en la práctica

- Habilidades de investigación 
En negrita se remarcan las más importantes, pero todas están representadas en mayor o menor grado en los objetivos didácticos de la actividad. En conjunto, ¿Piensas que la actividad consigue desarrollar aspectos de estos objetivos pretendidos?

Tabla 4. Resultados de la pregunta $n^{0} 4$

\begin{tabular}{lc}
\hline ¿Consigue el objetivo pretendido? & \% de estudiantes que eligen esta respuesta \\
\hline Lo consigue completamente & 39,39 \\
Lo consigue en su mayoría & 39,39 \\
Lo consigue sólo parcialmente & 21,21 \\
No lo consigue & 0,00 \\
\hline
\end{tabular}

PREGUNTA $n^{\circ}$ 5. Muchas veces los estudiantes pasan por las prácticas de un curso sin aprovecharlas realmente porque no hay un estudio previo de los métodos y protocolos que permita "entender bien lo que estoy haciendo" en el laboratorio. La actividad teórica "diseño del proyecto agua de fagos" se alinea con las prácticas de la asignatura de manera que pretenden que el estudiante inicie sus sesiones de laboratorio con una buena comprensión conceptual de los protocolos y del proyecto para entender los fundamentos y seguir adecuadamente las prácticas desarrolladas.

Tabla 5. Resultados de la pregunta $n^{0} 5$

\begin{tabular}{lc}
\hline ¿Consigue el objetivo pretendido? $\%$ de estudiantes que eligen esta respuesta \\
\hline Lo consigue completamente & 42,42 \\
Lo consigue en su mayoría & 39,39 \\
Lo consigue sólo parcialmente & 18,18 \\
No lo consigue & 0,00 \\
\hline
\end{tabular}

PREGUNTA $\mathrm{n}^{\circ}$ 6. Las prácticas siguen la metodología PBL (Problem-based learning). El objetivo es desarrollar autonomía y subir de nivel al estudiante en cuanto a su autoconfianza en su desempeño en un laboratorio real, su capacidad para entender protocolos experimentales, aplicar sus conocimientos teóricos a la práctica y ser capaz de resolver los problemas que suceden en el trabajo real en el laboratorio biotecnológico.

Tabla 6. Resultados de la pregunta $n^{0} 6$

\begin{tabular}{lc}
\hline ¿Consigue el objetivo pretendido? $\%$ de estudiantes que eligen esta respuesta \\
\hline Lo consigue completamente & 51,52 \\
Lo consigue en su mayoría & 30,30 \\
Lo consigue sólo parcialmente & 15,15 \\
No lo consigue & 3,03 \\
\hline
\end{tabular}


PREGUNTA $n^{\circ}$ 7. Uno de los objetivos más importantes de las prácticas es que el alumno aprenda a trasladar un protocolo desde la fuente original (manual de protocolos o artículo de investigación) a sus necesidades concretas dentro de su proyecto de investigación y las posibilidades de material y reactivos de su laboratorio. Una de las prácticas más formativas para ello es el adaptar desde un artículo el "ensayo de punto final para fagos".

Tabla 7. Resultados de la pregunta $n^{0} 7$

\begin{tabular}{lc}
\hline ¿Consigue el objetivo pretendido? & \% de estudiantes que eligen esta respuesta \\
\hline Lo consigue completamente & 51,52 \\
Lo consigue en su mayoría & 42,42 \\
Lo consigue sólo parcialmente & 3,03 \\
No lo consigue & 3,03 \\
\hline
\end{tabular}

Los resultados de la encuesta de evaluación son fundamentalmente muy positivos, no obstante, las preguntas $n^{\circ} 2$ y 3 , que se relacionan con la actividad escrita mediante mensajes en los foros de la plataforma Moodle, no son tan satisfactorios en cuanto a la acogida de estas actividades por parte de los alumnos.

A juzgar por la encuesta de evaluación, en opinión de los estudiantes la actividad consigue sus objetivos de aprendizaje en gran medida. No obstante, la realización de la fase teórica en grupos de debate en foros en la plataforma Moodle no ha sido bien acogida. Los alumnos prefieren reunirse de forma presencial antes que comunicar sus ideas por escrito.

Es habitual que los alumnos no realicen la importante preparación o estudio previo de la técnica o protocolo antes de introducirse en el laboratorio para su realización a nivel práctico. Incluso aunque dispongan del "manual de prácticas de laboratorio" con antelación, los estudiantes suelen omitir su estudio previo, tan importante para una buena asimilación y comprensión conceptual de la práctica que van a realizar. Un beneficio educativo del planteamiento del proyecto en dos fases diferenciadas, "teórica" y "práctica", es que el alumno llega a laboratorio con un dominio de las técnicas, protocolos y métodos inusitado respecto al habitualmente observado en prácticas anteriores de otras asignaturas. La elaboración del proyecto teórico ha forzado a una asimilación de los protocolos y conceptualización del proyecto práctico que redunda en un beneficio muy interesante: el alumno "sabe lo que hace" cuando trabaja en sus experimentos.

Los estudiantes experimentan una gran inseguridad en ciertos momentos del desarrollo del proyecto, la cual es una característica típica de la metodología ABP. Esta es más acusada durante la fase práctica, seguramente debido a que es la primera vez que se enfrentan a un trabajo práctico de laboratorio autónomo e independiente. En el desarrollo del proyecto escrito este efecto no es tan acusado, seguramente debido a que han desarrollado trabajos escritos bibliográficos en grupo en cursos anteriores. 
El profesor debe tenerlo muy en cuenta y observar y combatir la sensación de inseguridad que experimentan sus alumnos. Esta inseguridad se manifiesta de diversas formas.

Algunos estudiantes experimentan un bloqueo y permanecen pasivos sin ser capaces de iniciar ninguno de los experimentos previstos en la sesión. Ante esta situación, se recomienda que el instructor asista a estos estudiantes, animándoles insistentemente a realizar alguna tarea, y les proporcione guía e ideas para comenzar con el trabajo (“¿Cuál debe ser, en buena lógica, lo primero que debes obtener para empezar a trabajar con el proyecto?”; “¿En qué capítulo del libro de protocolos encontrarás seguramente la información que necesitas?"; “¿Cuántas muestras de tierra te interesa procesar, unas pocas o las más posibles?”).

Otros estudiantes reaccionan preguntando una y otra vez acerca de cualquier paso o fase del experimento que están desarrollando. Reclaman el papel o rol clásico del instructor, quieren que este les proporcione continúa asistencia o ayuda, tal como están acostumbrados por sus experiencias anteriores en sus prácticas en otras asignaturas. Este papel del instructor está absolutamente prohibido por la metodología ABP. El profesor debe abstenerse de resolver los problemas prácticos que se encuentran los alumnos porque es requisito para que se produzca el beneficio formativo esperado del ABP. Ahora bien, debido al limitado tiempo disponible, se propone una solución de compromiso. En estas situaciones, el profesor instará a los alumnos a que debatan entre ellos una posible solución al problema que les bloquea. Cuando obtengan por fin alguna posible idea, la comentarán con el profesor, que solo entonces les proporcionará la guía que necesitan para seguir avanzando. De esta manera, logramos el objetivo formativo del ABP (encontrar soluciones por uno mismo) sin comprometer el limitado tiempo disponible para el proyecto.

Durante las primeras sesiones, es normal que el instructor se encuentre sobrepasado por la gran cantidad de preguntas que recibe por parte de los estudiantes. También es frecuente que los grupos de alumnos detengan el progreso de su trabajo una y otra vez, a la espera de poder consultar con el instructor de prácticas la duda que les bloquea. En esta situación, el profesor debe instar a los alumnos que se hayan atascado en algún paso experimental que no detengan su trabajo, ya que hay múltiples tareas que pueden realizar para adelantar los experimentos posteriores (preparación de medios de cultivo, etc.) mientras llega su turno para consultar al instructor de prácticas.

El profesor debe ser en todo momento consciente de que está utilizando la metodología ABP, y que por tanto tiene prohibido intervenir o resolver dudas a sus estudiantes a no ser que lo considere estrictamente necesario. El proceso de aprendizaje basado en problemas trata de que los alumnos sean capaces de encontrar por sí mismos las respuestas a los problemas, preguntas y dudas que plantea la actividad ABP, por lo que el instructor debe evitar interferir con este proceso, ya que anularía el beneficio esperado de esta metodología docente. Sin embargo, los estudiantes no reciben bien que el profesor evite proporcionarles respuestas a sus dudas. Para evitar malentendidos y actitudes emocionalmente negativas hacia las prácticas e incluso hacia el propio instructor, se recomienda lo siguiente:

- el profesor explica todas las veces que considere conveniente o necesario a sus estudiantes el por qué tiene "prohibido resolver dudas en metodología ABP", ya que si lo hiciese impediría que el alumno persistiese en afrontar por sí mismo los problemas que se encuentra hasta encontrar sus propias soluciones, que es el mayor beneficio que ofrece al estudiante esta metodología 
- $\quad$ en cualquier caso, es muy conveniente tener preparadas una serie de ideas o frases de orientación para el alumno que pregunta que, sin proporcionar una respuesta directa, dirijan o enfoquen hacia una correcta actitud para que sean capaces de solventar por sí mismos sus propias dudas (por ejemplo "piensa en el proyecto, ¿qué producto final queréis conseguir obtener?" o "si tenéis dudas acerca de qué recipientes de entre los disponibles o qué volumen de muestra utilizar, pensad en ¿cuál va a ser más manejable para trabajar con comodidad? o ¿ese volumen será suficiente para disponer de producto en los pasos experimentales posteriores del proyecto?"

\section{Conclusiones}

En conclusión, creemos que la alineación de un trabajo escrito con las ulteriores prácticas de laboratorio rinde resultados muy positivos y consigue aprendizajes en los alumnos muy beneficiosos para la práctica profesional real y muy difíciles de conseguir mediante el diseño “clásico" de las prácticas de laboratorio basado en la repetición de la demostración del instructor y el seguimiento del "manual de prácticas de laboratorio".

\section{Referencias}

1. ALBANESE, M.A. Y MITCHELL S. (1993). Problem-based learning: a review of literature on its outcomes and implementation issues, Acad Med 68: 52-81

2. DINI, C (2011) Aislamiento y caracterización molecular de bacteriófagos de bacterias enteropatógenas para biocontrol de enfermedades transmitidas por alimentos (ETA). Tesis doctoral. Argentina. Centro de Investigación y Desarrollo en Criotecnología de Alimentos (CIDCA)

3. DUCH J., GROH S.E., ALLEN D.E. (Eds) (2001) The power of problem-based learning: a practical "how to" for teaching undergraduate courses in any discipline. Sterling Virginia US. Stylus Publishing.

4. KUTTER E, SULAKVELIDZE A (2004) Bacteriophages: Biology and Applications. CRC Press.

5. PRIETO, A., BARBARROJA, J., REYES, E., MONSERRAT, J., DÍAZ, D., VILLARROEL, M. Y ÁLVAREZMON, M. (2006). Un nuevo modelo de aprendizaje basado en problemas, el ABP 4x4. Aula Abierta, 87, 171-194.

6. SPRICIGO, DENIS AUGUSTO (2012). La Desinfección basada en bacteriófagos como herramienta de biocontrol de Salmonella en alimentos. Tesis doctoral. Barcelona. Departament de Genètica i de Microbiologia/ Universitat Autònoma de Barcelona. 